RAE-IC, Revista de la Asociación Española de Investigación de la Comunicación vol. 8, núm. 15 (2021), 2-23 ISSN 2341-2690

Recibido el 13 de diciembre de 2020 DOI: https://doi.org/10.24137/8.15.1 Aceptado el 20 de diciembre de 2020

\title{
La investigación post-disciplinaria como alternativa en comunicación
}

Post-disciplinary research as an alternative in communication

Fuentes Navarro, Raúl

Instituto Tecnológico y de Estudios Superiores de Occidente (ITESO)

raul@iteso.mx

Forma de citar este artículo:

Fuentes Navarro, R. (2020). La investigación post-disciplinaria como alternativa en comunicación. RAE-IC, Revista de la Asociación Española de Investigación de la Comunicación, 8(15), 2-23. https://doi.org/10.24137/8.15.1

\section{Resumen:}

Este artículo retoma trabajos previos del autor y los reformula para argumentar que hay indicios, cada vez más claros, de la adopción de modalidades "post-disciplinarias" en las prácticas institucionalizadas de producción de conocimiento sobre la comunicación en diversas regiones del mundo. Ante la creciente fragmentación y dispersión epistémica de este campo académico, y las transformaciones evidentes de las prácticas socioculturales que son sus referentes y objetos de estudio, la investigación postdisciplinaria puede representar una alternativa útil y consecuente con la historia misma de la institucionalización universitaria de esta especialidad, en la que convergen aportes de las humanidades y las ciencias sociales, con aparente independencia de las condiciones diferentes de los sistemas nacionales de educación superior. Se analizan 
algunas de las formulaciones más desarrolladas de esta perspectiva y sus implicaciones estratégicas para las prácticas universitarias en el campo.

Palabras clave: comunicación, campo académico, post-disciplinariedad.

\begin{abstract}
:
This paper takes up previous works by the author and reformulates them to argue that there are increasingly clear indications of the adoption of "post-disciplinary" modalities in the institutionalized practices of knowledge production on communication in various regions of the world. Faced with the growing epistemic fragmentation and dispersion of this academic field, and the evident transformations of the sociocultural practices that are its references and subject matters, post-disciplinary research may represent a useful alternative consistent with the very history of the university institutionalization of this specialty, in which contributions from the humanities and social sciences converge, with apparent independence from the different conditions of national higher education systems. Some of the more developed formulations of this perspective and their strategic implications for university practices in the field are analysed.
\end{abstract}

Keywords: communication, academic field, post-disciplinarity.

\title{
1. INTRODUCCIÓN
}

La meta-investigación histórica sobre las 'fronteras abiertas' de lo que la investigación internacional etiqueta como 'comunicación', contribuye a "hacernos más conscientes de los diversos, y a veces precarios, modos de institucionalización mediante los cuales se ha desarrollado el estudio de la comunicación pública, rompiendo los marcos académicos y profesionales más antiguos al tiempo que continuamos recurriendo a ellos" (Corner, 2019, p. 9).

Para los investigadores y practicantes de las ciencias de la comunicación en cualquier parte del mundo es bien sabido que identificar las fronteras de una disciplina científica, no se diga de un campo densamente multidisciplinario como este, es "un proceso dinámico y de alguna manera arbitrario, que requiere actualizaciones y refinamientos 
constantes" (Ioannidis et al., 2015, p. 4), así como una cuidadosa historización. La razón fundamental es que desde su escala más amplia, "el mundo del conocimiento está siendo transformado de un modelo centrífugo a un modelo centrípeto", según lo ha señalado Immanuel Wallerstein:

Desde mediados del siglo XIX hasta aproximadamente 1970, en el sistema universitario mundial hubo facultades separadas para las ciencias naturales y para las humanidades, que jalaban epistemológicamente en direcciones opuestas, con las ciencias sociales atrapadas en medio y desgarradas por esas dos poderosas fuerzas. Hoy tenemos científicos de la complejidad que usan un lenguaje más consonante con el discurso de la ciencia social (la flecha del tiempo) y representantes de los estudios culturales que hacen lo mismo (el anclaje social de los valores y los juicios estéticos), y ambos grupos están ganando fuerza. El modelo se está haciendo centrípeto en el sentido de que los dos extremos (la ciencia y las humanidades) se están moviendo en la dirección del polo central intermedio (la ciencia social) y en alguna medida en los términos de ese centro (Wallerstein, 2000, p. 31).

Este planteamiento sigue siendo útil veinte años después. Las ciencias de la comunicación, como las ciencias sociales en su conjunto, están desde su origen sujetas a esas tensiones y movimientos del "mundo del conocimiento", y además referidas a uno de los aspectos centrales y más cambiantes del mundo social. Por ello es indispensable reconocer y explorar las implicaciones no sólo de la emergencia y evolución de la "idea de comunicación" (Mattelart, 1995; Peters, 1999), sino las complejas circunstancias en que esta idea o ideas, han sido transmitidas en el tiempo y el espacio a sociedades distintas a aquellas donde se originaron, y donde necesariamente hay que recontextualizarlas, pues tienen sus historias propias (Beltrán et al., 2008) ${ }^{1}$. La meta-investigación de la comunicación parece ser, por ello, doblemente pertinente cuando se trabaja desde una región relativamente marginal en cuanto a la producción científica global, como Latinoamérica (Fuentes, 2018; 2019).

\footnotetext{
${ }^{1}$ Dos libros, a punto de aparecer publicados cuando se escribe este texto, prometen cubrir "Iagunas" enormemente influyentes en ese marco: por una parte el análisis comparativo y colaborativo entre investigadores europeos y latinoamericanos sobre seis tradiciones de investigación en comunicación, mediante el diálogo auspiciado conjuntamente por la Asociación Latinoamericana de Investigadores de la Comunicación (ALAIC) y la European Communication Research and Education Association (ECREA) (Paulino et al., Eds., en prensa); por otra, la conjunción de múltiples tradiciones y perspectivas teóricas de la comunicación bajo el concepto de "intervenciones globales" (Miike y Yin, Eds., forthcoming).
} 
Este artículo intenta sintetizar las líneas principales del trabajo avanzado por el autor sobre la meta-investigación histórica y la estructuración del campo académico de la comunicación, a partir de su estudio (heurístico) sobre el caso mexicano, desde una perspectiva sociocultural (Fuentes, 1998). Una primera sección recupera algunos de los marcos desde los cuales se ha construido y ubicado la problematización general sobre el campo; una segunda expone los contextos de la institucionalización académica, así como la ancestral y universal polémica sobre el carácter disciplinario de estos estudios; para finalmente desplegar en una tercera sección algunas maneras de interpretar y asumir los principios post-disciplinarios ${ }^{2}$ como estrategias de articulación universidadsociedad e investigación-formación profesional en el campo de estudios de la comunicación, con base en experiencias y debates concretamente situados.

\section{LAS PRÁCTICAS INSTITUCIONALIZADAS DE PRODUCCIÓN DEL CONOCIMIENTO}

La reflexión crítica sobre su propia estructuración institucional ha sido constante y universalmente desarrollada en el campo académico de la comunicación ${ }^{3}$. En la dimensión epistemológica, esta recurrencia ha llegado también a ser relativamente bien conocida en diversos ámbitos de las ciencias sociales e, incluso, incorporada como condición central en la formulación de la "nueva teoría social" (en el sentido de ruptura con el "consenso ortodoxo"), propuesta por Anthony Giddens en La Constitución de la Sociedad (1984), una obra en la que el autor intentó combinar, en el seno de una teoría de la estructuración, una doble sociología de las estructuras sociales y de la acción. El concepto de estructuración apunta en primer término "a hacernos percibir las estructuras sociales desde el ángulo del movimiento". Y aunque Giddens encaró de manera flexible los vínculos entre "conocimiento usual y conocimiento culto" del mundo social, el problema de que "las teorías de las ciencias sociales se entrelazan más o menos

\footnotetext{
2 El término "post-disciplinario", preferiblemente empleado como adjetivo y no como sustantivo, refiere a una condición de la práctica científica institucionalizada que rebasa, como se explicará más adelante, la estructura "normal" de las disciplinas, debido a la emergencia de fenómenos complejos, para cuya investigación y comprensión no bastan los recursos teórico-metodológicos de una disciplina. Por su referencia estructural y procesual, se prefiere sobre "transdisciplinario", aunque los sentidos son aproximadamente equivalentes.

3 La noción de "campo académico" ha sido desarrollada a partir de la obra sociológica de Pierre Bourdieu, entendida como "espacio" sociocultural de posiciones objetivas donde los agentes luchan por la apropiación del capital común, como uno de los conceptos centrales de las investigaciones del autor a partir de 1992, junto a la teoría de la estructuración de Giddens y el marco metodológico de la hermenéutica profunda de Thompson (Fuentes, 1998).
} 
con las teorías-en-uso de los actores" (Corcuff, 2013, pp. 60-62) implica un gozne conceptual y metodológico inevitablemente crítico y centrado en la comunicación.

En su obra teórica fundamental, en efecto, Giddens ubica en el postulado de la doble hermenéutica la condición esencial para que toda ciencia social sea inherentemente crítica: "las teorías y los hallazgos en las ciencias sociales es probable que tengan consecuencias prácticas (y políticas), independientemente de que el observador sociológico o el elaborador de políticas decidan que pueden ser 'aplicadas' a un asunto práctico particular" (Giddens, 1984, p. xxxv). De la misma manera, la doble hermenéutica identifica a la investigación social como una práctica sociocultural y comunicacional:

¿Qué es lo 'hermenéutico' en la doble hermenéutica? La propiedad del uso del término deriva del doble proceso de traducción o interpretación involucrado. Las descripciones sociológicas tienen la tarea de mediar los marcos de significado dentro de los cuales los actores orientan su conducta. Pero tales descripciones son categorías interpretativas que también requieren un esfuerzo de traducción para entrar y salir de los marcos de significado de las teorías sociológicas. (...) El científico social es un comunicador, al introducir marcos de significado asociados con ciertos contextos de la vida social a otros (Giddens, 1984, pp. 284-285).

Si bien las claves para evaluar y proyectar la articulación de los estudios académicos sobre la comunicación en términos de "campo" y de "disciplina" suelen ser epistemológicas en los hasta ahora interminables debates al respecto, hay también fuertes componentes sociológicos en juego, indispensables para problematizar e historizar los procesos de institucionalización de estos estudios, en la triple dimensión cognoscitiva, sociocultural e institucional (Fuentes, 1998, p. 48). Robert Craig ha desarrollado ampliamente, desde hace más de treinta años, su propuesta de considerar al estudio de la comunicación como una "disciplina práctica" (Craig, 1989; 1999; 2008), tomando como base precisamente la doble hermenéutica de Giddens, que responde a la condición de la ciencia social de interpretar hechos ya interpretados por los sujetos sociales y a la posibilidad de reintegrar los productos de la investigación en esos mismos marcos de interpretación. En la versión de Craig, "la comunicación como una disciplina 
práctica ha sido construida (incluso cuando reflexivamente lo reconstruye) sobre el fundamento de la comunicación como una categoría cada vez más central en las sociedades modernas y la cultura global" (Craig, 2008, p. 9).

Además de los múltiplemente referidos debates sobre el estudio de la comunicación y sus concreciones posibles como campo y/o disciplina, publicados recurrentemente en el Journal of Communication (1983, 1993, 2018), la necesidad de una revisión de sus condiciones y sus consecuencias aparece constantemente: casi todos los presidentes de la International Communication Association, por ejemplo, refrendan frecuentemente que ante las crisis generadas por cambios económicos, políticos y tecnológicos que afectan a todos los segmentos de la sociedad, y al campo de la comunicación, "nos debemos a nosotros mismos, a nuestros estudiantes y a nuestras sociedades enfrentar esos desafíos y repensar nuestra misión y nuestras prácticas de manera que contribuyan con soluciones" (Gross, 2012, p. 919).

También en ámbitos alejados de los centros estadounidenses, el cambio de marcos es una preocupación constante y prioritaria, especialmente ante la emergencia de sistemas digitales y prácticas novedosas de comunicación mediadas por ellos. Desde Australia, por ejemplo, John Hartley $(2012$, p. 3) subrayaba que, a su juicio, una cosa que los estudios mediáticos y culturales hacen particularmente bien "es estudiar el proceso situado y contextual ... de la emergencia de ideas en redes mediadas", y que entender "cómo se hace esto en una escala social amplia usando las últimas tecnologías de comunicación ya no es solo una cuestión de interés para los académicos de los medios y la cultura". Visto desde la región nórdica, según Kaarle Nordenstreng (2007, p. 212), el estudio de la comunicación sigue siendo más un campo que una disciplina, y "es una ilusión insana celebrar la popularidad de los estudios sobre medios con la distinción de una disciplina independiente, por no decir de varias disciplinas".

En Argentina, el Primer Coloquio de Comunicación para la Transformación Social convocado para celebrar el $40^{\circ}$ aniversario de la Escuela de Ciencias de la Información de la Universidad Nacional de Córdoba, declaró a su vez, entre sus puntos de partida, que el campo de la comunicación "es hoy un espacio multi y transdisciplinario de reflexión, reconstrucción y búsqueda de caminos alternativos y emergentes por lograr 
nuevas miradas, nuevos pensamientos, nuevas formas de vida. Es un ámbito de encuentros y discusiones, de aconteceres y críticas"4. Ahí conecta muy bien lo que, sin ser un consenso, es una posición crecientemente asumida en los debates científicos internacionales, que son siempre al mismo tiempo epistemológicos y políticos, según Bourdieu (2000, p. 15). La aceptación de una multiplicidad de perspectivas, que no necesariamente se oponen entre sí, y la dificultad de articularlas productivamente, es una condición bien establecida ante la incertidumbre y opacidad del cambio social. Y ya Thomas Kuhn (1982) señalaba hace décadas que la "tensión esencial" en la historia de la ciencia, la que produce su progreso, es la que se establece entre tradición y cambio.

Otras manifestaciones de estos debates, extendidos por el mundo durante la última década (Fuentes, 2014), se pueden encontrar en la obra de líderes del pensamiento sociológico internacional como el estadounidense Craig Calhoun (2011); el portugués Boaventura de Sousa Santos y su Epistemología del Sur (2009); o el francés Michel Wieviorka (2011). También en el Informe de la UNESCO sobre las Ciencias Sociales en el Mundo, de 2010, donde se documentan y analizan, con bastante claridad y desde perspectivas diversas, "las brechas del conocimiento" en el área 5 .

En términos generales, al analizar las tendencias recientes en el plano internacional, algunos observadores asumían en 2010 que las ciencias sociales pronto entrarían "en una era post-disciplinaria". Esa modalidad de organización académica implica fundamentalmente que la investigación se diseñe, ejecute y evalúe en función de "problemas de conocimiento situados" antes que de premisas y protocolos teóricometodológicos tradicionales, los que necesariamente resultan a su vez transformados en el proceso. Ahora, dependiendo de los autores, este cambio podría ser el causante de una nueva integración de las ciencias sociales y las naturales, o podría significar que el conocimiento estará cada vez más orientado hacia "comunidades epistémicas

\footnotetext{
${ }^{4}$ http://www.eci.unc.edu.ar/coloquio40/, consultado el 24/08/2012 y el 21/12/2013, ya no disponible en el sitio de la ECl el $10 / 12 / 2020$.

${ }^{5}$ Por una parte, en cuanto a la "geografía institucional", pues "las diferencias entre regiones y países en el estatus de la investigación de las ciencias sociales no podrían ser mayores" (UNESCO, 2012, p. 53); por otra, en cuanto a la distribución lingüística de los productos de esa investigación: no obstante que el español y el portugués son respectivamente la cuarta y la quinta lenguas más usadas en el mundo (superadas solamente por el inglés, el francés y el alemán) entre las revistas indizadas en ciencias sociales, según indicadores de Ulrich y de Thomson-SSCl, la proporción de la producción mundial que difunden es mínima, pues en conjunto alcanzan apenas entre el 0.5 y el 5.7 por ciento mundial, dependiendo de los períodos y los métodos de medición (UNESCO, 2012, p. 155).
} 
integradas", cuyo interés sea la solución de problemas locales y contextuales (UNESCO, 2012, p. 197), con el riesgo, ya presente, de la fragmentación. Y en esta perspectiva postdisciplinaria, que formula quizá la vertiente más interesante del impulso para "abrir las ciencias sociales" (Wallerstein et al., 1996), los estudios de comunicación aparecen, con naturalidad y dignidad, en muchos de los recuentos y revisiones del estado de las ciencias sociales, como referencia de la transformación en curso de los "territorios disciplinarios". Se puede leer en el Informe de la UNESCO el conjunto de tensiones que esta referencia implica:

Los estudios de la comunicación [...] tienen algunos de los rasgos de un campo transdisciplinario e interdisciplinario; sin embargo, recientemente han adquirido mucha de la parafernalia institucional y profesional de una disciplina académica, incluyendo crecientes ofertas de cursos universitarios, lo cual resulta en un número mayor de académicos contratados, departamentos en universidades, asociaciones profesionales nuevas y conferencias. Actualmente 'comunicación' es identificada como una categoría separada en las bases de datos bibliográficas de ciencias sociales como el SSCI Thomson Reuters, y el número de artículos publicados en esta categoría muestra una tendencia a aumentar. Incluso esto puede no reflejar el número aún mayor de libros de texto publicados anualmente en este campo (UNESCO, 2012, p. 204).

A pesar de la fuerza política y económica empleada por algunos gobiernos iberoamericanos para impulsar una institucionalización "productiva, vinculada, e internacional" de la ciencia y la educación superior, las tensiones organizacionales siguen siendo clave para el desarrollo de las muy pobladas ciencias sociales. Por ello, una pregunta metodológica que el propio Informe de la UNESCO formula en relación con los "cruces" de las fronteras disciplinarias en ciencias sociales podría ser, quizá, mejor respondida atendiendo a la experiencia de los estudios de comunicación: “¿Cómo puede ser fortalecida la formación interdisciplinaria mientras que las disciplinas se fortalecen? Esta puede ser la pregunta práctica de mañana para la investigación en ciencias sociales" (UNESCO, 2012, p. 213). 


\section{CAMPOS CIENTÍFICO, EDUCATIVO, PROFESIONAL}

A partir de opciones teórico-metodológicas tomadas para orientar la búsqueda de respuestas a las preguntas centrales de la investigación sobre la constitución del campo académico de la comunicación en México (Fuentes, 1998, pp. 68-71), se decidió la formulación de dos modelos heurísticos. El primero de ellos ("estructural"), tuvo como propósito distinguir (y relacionar entre sí) tres modalidades de prácticas académicas: las centradas en la producción, en la reproducción y en la aplicación del conocimiento sobre la comunicación. El supuesto de este modelo es que cada una de estas modalidades está sujeta a diversas determinaciones (tanto internas como externas), y que tendrían que estar articuladas entre sí mediante un núcleo común de sentido básico compartido, que constituiría lo que podría llamarse "matriz disciplinaria", mejor que "paradigma" (Kuhn, 1982).

Las prácticas de producción de conocimiento se engloban bajo el término "investigación", cuya institucionalización se considera escindida en dos vertientes: la académica y la aplicada. Aunque la primera (realizada en las universidades, sujeta a las normas de la actividad académico-científica, y por tanto pública en su financiamiento, objetivos, procedimientos y resultados) es la enfocada en el trabajo, no puede ignorarse la segunda (realizada generalmente por empresas especializadas, sujeta a las leyes del mercado de prestación de servicios y, por tanto, privada, confidencial o hasta secreta en su financiamiento, objetivos, procedimientos y resultados). En sus dos vertientes, las prácticas de investigación se realizan como concreciones de marcos lógicos, ideológicos, técnicos y éticos de las ciencias sociales, a los cuales realimentan.

Las prácticas de aplicación del conocimiento se centran en el ámbito general de la profesión que opera los sistemas de comunicación social y son reguladas por el mercado en que concurren tanto las instituciones especializadas en esta función (medios "masivos", agencias de publicidad o de noticias, etc.), como individuos calificados formal (título universitario, colegio o asociación profesional) o informalmente (experiencia reconocida) como competentes en alguna de las múltiples especialidades de esta rama de la actividad económica. Las prácticas profesionales en las industrias culturales han 
sido el referente primordial del campo académico, especialmente en su modalidad reproductiva de los saberes instrumentales que las constituyen.

Las prácticas de reproducción del conocimiento y de los agentes que lo portan operativamente en el campo de la comunicación, consideradas en tanto "formación profesional", son las que median desde las universidades la conformación del campo en términos socioculturales. Para hacer esto, las prácticas académicas articulan los planos científico y profesional, mediante programas institucionales de docencia e investigación. En este punto se considera la tradicional definición de las "funciones sustantivas" de la institución universitaria (la formación profesional o docencia superior, la investigación científica y humanística y el servicio o extensión universitaria) y su integración, como determinantes de la inserción (función) social concreta de cada institución.

Los modos y grados de articulación del campo académico (entre las prácticas de "investigación", "profesionales" y de "formación", que a su vez se estructuran en los subcampos científicos, profesionales y educativos), sirven como parámetros de contrastación externa de la estructuración consistente del campo académico, al proporcionar indicios de su ajuste a las condiciones de desarrollo de las prácticas (y las agencias) sociales que toma como objetos, y en consecuencia, al otorgar reconocimiento y legitimidad en grados variables a las prácticas académicas diferencialmente institucionalizadas.

Pero es específicamente en los modos y grados de articulación entre los subcampos científico y educativo (y entre las prácticas de investigación y de formación profesional), donde se ubican los parámetros de la consistencia interna de la estructuración del campo académico, mediante una matriz disciplinaria, que consistiría no sólo en "generalizaciones, modelos y ejemplares" como estableció Kuhn (1982, p. 321), sino también y sobre todo, en esquemas interpretativos, en una ideología profesional específica, conformada por sistemas de significación, de valoración (reglas morales y éticas) y de recursos de poder, que son el referente estructural del habitus y de la agencia de los sujetos. 
El segundo modelo heurístico de aquel estudio, centrado en los "procesos de estructuración" del campo, operantes en tres escalas (individual, institucional y sociocultural) y tres dimensiones (económica, política, cultural) interrelacionadas, buscó distinguir y articular entre sí los procesos de constitución de los sujetos, la formación/conformación del habitus, la profesionalización, la institucionalización social y la cognoscitiva, la especialización de la producción, la auto-reproducción del campo, su legitimación social y la asimilación/acomodación del sentido en el entorno sociocultural (Fuentes, 1998, pp. 71-75). Para Craig (2008, pp. 8-9), concurrentemente, son tres los contextos histórico-sociales en los que se construye y de los que proviene la legitimidad del campo: contextos intelectuales, institucionales y socioculturales ${ }^{6}$.

Entre las múltiples fuentes científicas de interés para la contrastación de estos postulados, está la obra del sociólogo de la Universidad de Chicago Andrew Abbott, que ha elaborado interesantes explicaciones sobre la dinámica histórica de las disciplinas (1988) y algunas profesiones (2001) en Estados Unidos, basadas en el concepto de "campos de interacción", parcialmente tomado de Bourdieu, así como sobre los métodos heurísticos en ciencias sociales (2004). Abbott señala muy bien la particularidad del sistema académico estadounidense, en comparación con los sistemas tradicionales alemán, francés y británico, donde no pueden, en sentido estricto, reconocerse estructuras disciplinarias, al menos hasta antes de las recientes reformas unificadoras de las universidades europeas. Y sobra mencionar las radicales diferencias estructurales con los sistemas latinoamericanos.

Para Abbott, el sistema académico estadounidense, en el que la estructura de las disciplinas académicas se estableció entre fines del siglo XIX y principios del XX, obtiene su fuerza y estabilidad de una "doble institucionalización": por un lado, un mercado laboral interuniversitario regulado por las asociaciones disciplinarias que mantiene en movimiento constante a los académicos, y por el otro una organización curricular intrauniversitaria centrada en la figura de los "majors", controlada por los

\footnotetext{
${ }^{6} \mathrm{Y}$ esa triple dimensionalidad ha sido también asumida, en términos equiparables, por investigadores de distintos países y regiones (Koivisto y Thomas, 2008; Park y Pooley, 2008; Rodrigo y García, 2010; Calhoun, 2011; Löblich y Scheu, 2011; Moragas, 2011; Stanfill, 2012; Craig, 2015; Gehrke y Keith, 2015; Simonson y Park, 2016; Löblich y Averbeck-Lietz, 2016; Averbeck-Lietz, 2017. En América Latina, Marques de Melo, 1992, 1998, 2007; Fuentes, 1992, 1998, 2006; Orozco, 1997; Bolaño, Crovi y Cimadevilla, 2015; Crovi Coord., 2018; Crovi y Cimadevilla, 2018; Crovi y Trejo, 2018).
} 
departamentos, que "disciplina anualmente a millones de estudiantes" (Fuentes, 2014, pp. 94-95).

Pero la historia del estudio de la comunicación, como todas las demás perspectivas de análisis y reflexión relacionadas en este campo (teoría, epistemología, metodología, ontología), admite diferentes versiones, algunas enfrentadas entre sí y otras simplemente coexistentes. La diversidad de objetos de conocimiento que se han agrupado bajo el término "comunicación" y la diversidad de disposiciones institucionales para organizar su estudio académico en distintos países y regiones, se han convertido en centro de atención para las secciones de Historia de asociaciones académicas internacionales (como la International Communication Association, ICA, y la International Association for Media and Communication Research, IAMCR), de donde han surgido contribuciones influyentes para la meta-investigación del propio campo. Si bien "hasta ahora, la mayor parte de las historias han sido nacionales, con una predominante atención sobre América del Norte y Europa Occidental" (Simonson y Peters, 2008, p. 764), se ha fortalecido recientemente una perspectiva que "nos ayuda a ver cómo el estudio organizado de la comunicación al mismo tiempo ha reflejado, refractado e impulsado la geopolítica transnacional, los patrones institucionales de educación y profesionalización y maneras de conocer y de actuar" determinantes de la vida colectiva (Pooley y Park, 2013, pp. 85-86).

Pero los cuestionamientos fundamentales que la meta-investigación histórica permite atender cada vez más analíticamente y responder de maneras cada vez más convincentes, han estado y siguen presentes a todo lo largo de la historia de las ciencias de la comunicación, sobre todo en relación con su identidad teórica, en distintas épocas, regiones y lenguas (Pasquali, 1978; Verón, 1987; Martín-Barbero, 1987; Craig, 1999; Curran y Park, 2000; Martín-Serrano, 2007; Krippendorff, 2009; Chung, 2013; Sodré, 2014; Vizer y Vidales, 2016). Muchos de estos y otros aportes a la meta-investigación de la comunicación fueron sistemáticamente revisados por el británico John Corner en un artículo reciente, en el que se destaca en particular la expansión del trabajo histórico sobre el estudio de la comunicación para incluir contextos internacionales y "el examen sobre cómo han contribuido los programas de enseñanza y la actividad de investigación 
a institucionalizar el área con una discreta, aunque muy debatida, identidad académica" (Corner, 2019, p. 1). Los tres factores que subraya Corner son los diversos contextos donde se originaron los programas, los vínculos históricos de la investigación con las prácticas profesionales y el impacto de los nuevos medios en la historia reciente tanto de la enseñanza como de la investigación.

\section{ESTRATEGIAS UNIVERSITARIAS ¿INTERDISCIPLINARIAS O POST-DISCIPLINARIAS?}

A partir, sobre todo, de la "irrupción de Internet" en las prácticas socioculturales más generalizadas de comunicación, a mediados de los años noventa (Fuentes, 2001), fue notable el cambio que pudo percibirse en muchos de los objetos de estudio de las ciencias de la comunicación, así como en los marcos teóricos y metodológicos disponibles para abordarlos, por lo que en el mundo entero y en el conjunto de las ciencias sociales, se multiplicaron las estrategias "interdisciplinarias", tanto en la investigación como en la formación consecuente de investigadores. Además de un desafío abstracto, la interdisciplinariedad se convirtió en un recurso, y en un problema, de carácter práctico, asociados a la expansión de centros de investigación y de académicos de tiempo completo vinculados a proyectos. Entendida como una estrategia de formación universitaria y de ejercicio de la investigación, la interdisciplinariedad se convirtió en una experiencia a confrontar, una práctica social a interpretar, un imperativo a problematizar, todo lo cual es una tarea colectiva, intersubjetiva e interinstitucional muy concretamente situada.

Pero como es obvio, para diseñar y evaluar estrategias interdisciplinarias, hay que partir de qué son las disciplinas. Immanuel Wallerstein (2005) afirma que son tres cosas al mismo tiempo: son categorías intelectuales, que sirven para construir objetos de estudio; son estructuras institucionales, que organizan tanto la práctica de la investigación como la formación especializada, y también sirven para distribuir presupuestos y legitimar proyectos. Finalmente, las disciplinas son culturas, modos de ser académicos, sustentos de identidades, conjuntos de supuestos y de estilos de pensamiento que fomentan la discusión y la colaboración, el avance y la consolidación. Cuando se habla de "interdisciplinariedad", es necesario especificar las implicaciones que la relación entre disciplinas supone en estas tres dimensiones. 
Pero también, sobre otro eje, hay que reconocer que las disciplinas cumplen dos funciones interrelacionadas, que complejizan aún más el debate y la práctica de la interdisciplinariedad: por un lado, la disciplina es un modo específico de proceder que articula a la comunidad de especialistas, que es otro nombre para su identidad, que sirve para distinguirla de otras y para legitimarla, en su triple carácter intelectual, institucional y sociocultural. Y por otro lado, donde siguen vigentes las acepciones religiosa y militar originales del verbo "disciplinar", está la disciplina que se enseña a los aspirantes o se impone a los discípulos, que se transmite de generación en generación para reproducir no sólo el modo específico de proceder en la producción académica, sino también de relacionarse con el mundo social externo a la comunidad, de preservar e intensificar la identidad ${ }^{7}$.

Hay que considerar que, al menos en los campos de las ciencias sociales y las humanidades, aunque también y quizá con mayor profundidad en los campos de investigación de las ciencias naturales, las estrategias interdisciplinarias son un hecho establecido, una práctica cada vez más común, y un grave problema para los sistemas de clasificación y evaluación institucionalizados. Puede mantenerse, no obstante, la certeza de que no hay mejor manera de aprender a hacer investigación interdisciplinaria que haciéndola, y sobre todo, haciéndola reflexivamente. Por eso, antes que por las razones de legitimidad simbólica y distinción social, que por supuesto cuentan mucho también, es que la formación universitaria mantiene su justificación histórico-social. Y por eso es relevante cuestionar la eficacia educativa, la articulación entre los procesos de formación universitaria y la inserción socioprofesional de los egresados.

Por otra parte, desde la perspectiva de las humanidades, el académico estadounidense Louis Menand contrastaba hace unos años dos tendencias diferentes en la educación superior de su país: una, la "interdisciplinaria", que supone el fortalecimiento de las disciplinas, sobre todo en su aspecto institucional; otra, la "post-disciplinaria", caracterizada por el creciente eclecticismo metodológico y temático en la investigación y la enseñanza. Para él, "la enseñanza o la producción académicas interdisciplinarias

\footnotetext{
${ }^{7}$ Es necesario precisar que el concepto de "disciplina" aquí mencionado, sigue una acepción sociológica y educacional que no implica la discusión más profunda, que corresponde a otro lugar, de la tradición foucaultiana (Foucault, 1976) y otras, aunque está obviamente relacionada.
} 
simplemente significan el despliegue de la experticia profesional en dos o más disciplinas, y este fenómeno no es lo mismo que la post-disciplinariedad", que tiene más que ver con la construcción de nuevos objetos de conocimiento de maneras nuevas, que las disciplinas tradicionales son incapaces de producir (Menand, 2010, p. 87). La postura de Menand no es favorable ante este cambio en el horizonte académico, y aunque no menciona explícitamente a los estudios de comunicación en su crítica de los cambios en la educación superior estadounidense, la versión de 2001 de su texto termina con una advertencia relacionada directamente con la "cultura" comunicada, en las universidades y por los medios, que es pertinente revisar, sobre todo por el riesgo que entraña la pérdida de los parámetros de rigor académico:

Algo que no ha cambiado es la delicada y en cierto sentido paradójica relación entre la universidad y la cultura general. Es importante que la investigación y la enseñanza sean relevantes, para que la universidad se involucre con la cultura pública y para diseñar sus paradigmas investigativos teniendo en cuenta la vida social y cultural real. [...] Para seguir siendo relevante hoy, creo que la indagación académica tiene que volverse menos especializada, menos técnica, menos excluyente y más holística. Espero que ese sea el camino al que nos lleve la post-disciplinariedad. Al final de este camino, no obstante, hay un gran riesgo, que es el que la cultura de la universidad se convierta en nada más que un eco de la cultura pública. Eso sería una catástrofe. La tarea académica en una sociedad libre es servir a la cultura pública haciendo las preguntas que el público no quiere formular, investigando los asuntos que otros no pueden o no quieren investigar, haciendo espacio a las voces que no tienen lugar o que son rechazadas. Los académicos necesitan mirar el mundo para ver qué clases de enseñanza y de pensamiento tienen que elaborarse, y cómo pueden organizarse mejor para hacerlo, pero necesitan ignorar la insistencia del mundo para que reproduzcan su imagen (Menand, 2001, s/p).

Y esa advertencia puede servir para cerrar esta revisión de la emergencia de la "postdisciplinariedad" en el campo académico de la comunicación, reconocida desde hace muchos años (Fuentes, 1994) pero todavía incipientemente asumida, y recomendar la lectura y debate de, al menos, dos libros donde se desarrolla muy seriamente y se argumenta desde dos contextos académicos y sociales diferentes aunque eventualmente convergentes: el brasileño en A Ciência do Comum de Muniz Sodré 
(2014) (traducido al inglés como The Science of the Commons en 2019), y el estadounidense en Communication: A Post-Discipline, de Silvio Waisbord (2019). El primero enfatiza la constitución de un "método comunicacional" para la "organización de lo común" a partir del "bios virtual" o "mediático". El segundo la "construcción de puentes" entre lo teórico y lo analítico, para impulsar una "imaginación comunicativa", paráfrasis de la Imaginación Sociológica de C. Wright-Mills (1959). Ambos son ejemplos de erudita y lúcida reflexión sobre el estudio de la comunicación y sus alternativas de reconstitución crítica y socialmente comprometida desde una práctica académica postdisciplinaria, que merecen ser debatidos desde todas las perspectivas involucradas en el campo.

\section{REFERENCIAS BIBLIOGRÁFICAS}

Abbott, A. (1988). The System of Professions. An essay on the division of Expert Labor. Chicago, London: The University of Chicago Press.

Abbott, A. (2001). Chaos of Disciplines. Chicago, London: The University of Chicago Press.

Abbott, A. (2004). Methods of discovery: heuristics for the social sciences. New York: W.W. Norton \& Co.

Averbeck-Lietz, S. (Ed.) (2017). Kommunikationswissenschaft im internationalen vergleich. Wiesbaden: Springer.

Beltrán, L. R. et al. (2008). La Comunicación antes de Colón. Tipos y formas en Mesoamérica y los Andes. La Paz: Centro Interdisciplinario Boliviano de Estudios de la Comunicación.

Bolaño C., D. Crovi y G. Cimadevilla, (Coords.) (2015). La contribución de América Latina al campo de la comunicación. Historia, enfoques teóricos, epistemológicos y tendencias de la investigación. Buenos Aires: Prometeo.

Bourdieu, P. (2000). El campo científico, en Los usos sociales de la ciencia. Buenos Aires: Nueva Visión. 
Calhoun, C. (2011). Communication as Social Science (And More), International Journal of Communication, 5, 1479-1496. Recuperado de http://ijoc.org/index.php/ijoc/article/view/1331/622

Chung, J. C. et al. (2013). An analysis on communication theory and discipline. Scientometrics, 95, 985-1002. https://doi.org/10.1007/s11192-012-0869-4

Corcuff, P. (2013). Las nuevas sociologías. Principales corrientes y debates, 1980-2010. Buenos Aires: Siglo Veintiuno editores.

Corner, J. (2019). Origins and transformations: histories of communication study. Media, Culture \& Society, 41(5), 1-11. https://doi.org/10.1177/0163443718820666

Craig, R. T. (1989). Communication as a practical discipline. En B. Dervin, L. Grossberg, B. O'Keeffe y E. Wartella (Eds.), Rethinking Communication. Volume I: Paradigm Issues (pp. 97-122). Newbury Park, CA: Sage.

Craig, R. T. (1999). Communication Theory as a Field. Communication Theory, 9(2), 119-161. https://doi.org/10.1111/j.1468-2885.1999.tb00355.x

Craig, R. T. (2008). Communication in the conversation of disciplines. Russian Journal of Communication, 1(1), 7-23. https://doi.org/10.1080/19409419.2008.10756694

Craig, R. T. (2015). The Constitutive Metamodel: A 16-year review. Communication Theory, 25(4), 356-374. https://doi.org/10.1111/comt.12076

Crovi, D. (Coord.) (2018). Sociedad del conocimiento y comunicación. Reflexiones críticas desde América Latina. México: ALAIC.

Crovi, D. y G. Cimadevilla (Coords.). (2018) Del mimeógrafo a las redes digitales. Narrativas, testimonios y análisis del campo comunicacional en el 40 aniversario de ALAIC. México: ALAIC.

Crovi, D. y Trejo, R. (Coords.) (2018). Tejiendo nuestra historia. Investigación de la comunicación en América Latina. México: UNAM. 
Curran, J. y Park M.-J. eds. (2000). De-Westernizing Media Studies. London, New York: Routledge.

de Moragas, M. (2011). Interpretar la comunicación. Estudios sobre medios en América y en Europa. Barcelona: Gedisa.

Foucault, M. (1976). Vigilar y castigar. Nacimiento de la prisión. México: Siglo XXI.

Fuentes N., R. (1992). Un campo cargado de futuro. El estudio de la comunicación en América Latina. México: FELAFACS.

Fuentes N., R. (1994). La investigación de la comunicación ¿hacia la postdisciplinariedad en ciencias sociales? En J. Lameiras y J. Galindo (Eds.), Medios y Mediaciones (pp. 221-243). Guadalajara: El Colegio de Michoacán/ ITESO.

Fuentes N., R. (1998). La emergencia de un campo académico: continuidad utópica y estructuración científica de la investigación de la comunicación en México. Guadalajara: ITESO y Universidad de Guadalajara.

Fuentes N., R. (2001). Exploraciones teórico-metodológicas para la investigación sociocultural de los usos de Internet. En M. I. Vassallo de Lopes y R. Fuentes Navarro (Comps.), Comunicación, campo y objeto de estudio. Perspectivas reflexivas latinoamericanas (pp. 229-245).. Guadalajara: ITESO/ U.A.A./ U. de Colima/ U. de Guadalajara.

Fuentes N., R. (2006). Para documentar las relecturas y reescrituras del campo latinoamericano de investigación de la comunicación. En R. Fuentes (Coord.), Instituciones y redes académicas para el estudio de la comunicación en América Latina (pp. 183-226). Guadalajara: ITESO.

Fuentes N., R. (2014). Comunicação e dupla hermenêutica: convergências entre disciplinas científicas e profissões. MATRIZes, 8(2), 79-100.

http://dx.doi.org/10.11606/issn.1982-8160.v8i2p79-100

Fuentes N., R. (2018). Tendencias regionales y transnacionales de la investigación de la comunicación en América Latina. En D. Crovi y R. Trejo (Coords.) Tejiendo nuestra 
historia. Investigación de la comunicación en América Latina (pp. 295-315). México: UNAM.

Fuentes N., R. (2019). Pesquisa e meta-pesquisa sobre comunicação na América Latina. MATRIZes, 13(1), 27-48. http://dx.doi.org/10.11606/issn.1982-8160.v13i1p27-48

Gehrke, P. J. y Keith, W. M. (Eds.) (2015). A Century of Communication Studies: The Unfinished Conversation. London; New York: Routledge.

Giddens, A. (1984). The Constitution of Society. Outline of the Theory of Structuration. Berkeley/Los Angeles: University of California Press.

Gross, L. (2012). Fastening Our Seatbelts: Turning Crisis into Opportunity. Journal of Communication, 62, 919-931. https://doi.org/10.1111/j.1460-2466.2012.01679.x Hartley, J. (2012). Digital Futures for Cultural and Media Studies. UK: Wiley-Blackwell. Ioannidis, J. P. A., et al. (2015). Meta-research: Evaluation and Improvement of Research Methods and Practices. PLoS Biol 13(10), e1002264. https://doi.org/10.1371/journal.pbio.1002264

Koivisto, J. et al. (2008). Mapping Media and Communication Research: Paradigms, Institutions, Challenges. Helsinki: Communication Research Center, University of Helsinki.

Krippendorff, K. (F. Bermejo, Ed.) (2009). On Communicating: Otherness, Meaning, and Information. New York, London: Routledge.

Kuhn, T. S. (1982). La tensión esencial, estudios selectos sobre la tradición y el cambio en el ámbito de la ciencia. México: CONACYT/ Fondo de Cultura Económica.

Löblich, M. y Scheu, A. M.(2011). Writing the History of Communication Studies: A Sociology of Science Approach. Communication Theory, 21(1), 1-22. https://doi.org/10.1111/j.1468-2885.2010.01373.x

Löblich, M. y Averbeck-Lietz, S. (2016). The transnational flow of ideas and histoire croisée with attention to the cases of France and Germany. En P. Simonson y D. Park 
(Eds.), The International History of Communication Study (pp. 25-46). New York, London: Routledge.

Marques de Melo, J. (Coord.) (1992). Comunicación Latinoamericana: Desafíos de la Investigación para el Siglo XXI. São Paulo: ECA USP / ALAIC.

Marques de Melo, J. (1998). Teoria da Comunicação: paradigmas latino-americanos. Petrópolis: Vozes.

Marques de Melo, J. (2007). Entre el saber y el poder. Pensamiento comunicacional latino-americano. Monterrey: Comité Regional Norte de Cooperación con la UNESCO. Martín-Barbero, J. (1982). Retos a la investigación de comunicación en América Latina. Comunicación y Cultura, 9, 99-114.

Martín-Barbero, J. (1987). De los Medios a las Mediaciones. Comunicación, Cultura y Hegemonía. Barcelona: Gustavo Gili.

Martín-Serrano, M. (2007). Teoría de la Comunicación. La comunicación, la vida y la sociedad. Madrid: McGraw Hill Interamericana.

Mattelart, A. (1995). La invención de la comunicación. México: Siglo XXI.

Menand, L. (2001). The Marketplace of Ideas. American Council of Learned Societies Occasional Paper, 49. Recuperado de http://archives.acls.org/op/49_Marketplace_of_Ideas.htm

Menand, L. (2010). The Marketplace of Ideas. New York, London: W.W. Norton \& Co. Miike, Y. y Yin, J. (Eds.) (en prensa). Handbook of Global Interventions in Communication Theory. New York: Taylor \& Francis/Routledge.

Nordenstreng, K. (2007). Discipline or Field? Soul-searching in Communication Research. Nordicom Review, 28, pp.211-222. Recuperado de http://urn.fi/URN:NBN:fi:uta-201511242438 
Orozco G., G. (1997). La investigación de la comunicación dentro y fuera de América Latina. Tendencias, perspectivas y desafíos del estudio de los medios. La Plata: Universidad Nacional de La Plata.

Park, D. W. y Pooley, J. (Eds.) (2008). The History of Media and Communication Research: Contested Memories. New York: Peter Lang.

Pasquali, A. (1978). Comprender la Comunicación. Caracas: Monte Ávila.

Paulino, F. O. et al. (Eds.) (en prensa). Tradiciones de investigación en diálogo. Estudios sobre Comunicación en América Latina y Europa. Porto: Media XXI Publishing.

Peters, J. D. (1999). Speaking into the Air: A History of the Idea of Communication. Chicago, London: The University of Chicago Press.

Pooley, J. D. y Park, D. W. (2013). Communication Research. En P. Simonson, J. Peck, R. Craig y J. Jackson Jr. (Eds.), The Handbook of Communication History (pp. 76-90). New York, London: Routledge.

Rodrigo, M. y García, L. (2010). Communication theory and research in Spain: A paradigmatic case of a socio-humanistic discipline. European Journal of Communication, 25(3), 273-286. https://doi.org/10.1177/0267323110373458

Santos, B. de S. (2009). Una Epistemología del Sur: la reinvención del conocimiento y la emancipación social. México: Siglo Veintiuno/ CLACSO.

Simonson, P. y Peters, J. D. (2008). Communication and Media Studies, History to 1968. En W. Donsbach (Ed.), The International Encyclopedia of Communication Vol. II (pp.764-771). New York: Blackwell.

Simonson, P. y Park, D. W. (Eds.) (2016). The International History of Communication Study. New York, London: Routledge.

Sodré, M. (2014). A Ciência do Comum. Notas para o método comunicacional. Petrópolis: Vozes. 
Stanfill, M. (2012). Finding Birds of a Feather: Multiple Memberships and Diversity without Divisiveness in Communication Research. Communication Theory, 22, 1-24. http://dx.doi.org/10.1111/j.1468-2885.2011.01395.x

UNESCO (2012). Informe sobre las Ciencias Sociales en el Mundo. Las brechas del conocimiento. México: UNESCO/ISSC/ Foro Consultivo Científico y Tecnológico/ COMECSO.

Verón, E. (1987). La Semiosis Social. Fragmentos de una teoría de la discursividad. Barcelona: Gedisa.

Vizer, E. y Vidales, C. (Coords.) (2016). Comunicación, campo(s), teorías y problemas. Una perspectiva internacional. Salamanca: Comunicación Social.

Waisbord, S. (2019). Communication: A Post-Discipline. Cambridge UK: Polity Press. Wallerstein, I. et al. (1996). Abrir las Ciencias Sociales. México: Siglo XXI/ CIIH UNAM. Wallerstein, I. (2000). From sociology to historical social science: prospects and obstacles, British Journal of Sociology, 51(1), 25-35. https://doi.org/10.1111/j.14684446.2000.00025.x

Wallerstein, I. (2005). Las incertidumbres del saber. Barcelona: Gedisa.

Wieviorka, M. (2011). Una Sociología para el Siglo XXI. Barcelona: UOC Ediciones. Wright-Mills, C. (1959). The Sociological Imagination. New York: Oxford. 